\title{
CONNECTED ALGEBRAIC MONOIDS ${ }^{1}$
}

\author{
BY
}

MOHAN S. PUTCHA

\begin{abstract}
Let $S$ be a connected algebraic monoid with group of units $G$ and lattice of regular $\mathcal{G}$-classes $\mathcal{U}(S)$. The connection between the solvability of $G$ and the semilattice decomposition of $S$ into archimedean semigroups is further elaborated. If $S$ has a zero and if $|\mathcal{Q}(S)| \leqslant 7$, then it is shown that $G$ is solvable if and only if $\mathcal{Q}(S)$ is relatively complemented. If $J \in \mathcal{Q}(S)$, then we introduce two basic numbers $\theta(J)$ and $\delta(J)$ and study their properties. Crucial to this process is the theorem that for any indempotent $e$ of $S$, the centralizer of $e$ in $G$ is connected. Connected monoids with central idempotents are also studied. A conjecture about their structure is forwarded. It is pointed out that the maximal connected submonoids of $S$ with central idempotents need not be conjugate. However special maximal connected submonoids with central idempotents are conjugate. If $S$ is regular, then $S$ is a Clifford semigroup if and only if for all $f \in E(S)$, the set $\{e \mid e \in E(S), e \geqslant f\}$ is finite. Finally the maximal semilattice image of any connected monoid is determined.
\end{abstract}

Introduction. Let $K$ be an algebraically closed field. By a (linear) algebraic semigroup, we mean a Zariski closed subsemigroup of the multiplicative semigroup of all $n \times n$ matrices over a field. An algebraic semigroup is connected if the underlying closed set is an irreducible variety. Connected groups have been well studied [1]. In this paper we continue our study of connected monoids. Our basic model of a connected monoid is the multiplicative semigroup of all $n \times n$ matrices, $\mathfrak{N}_{n}(K)$. Even if one is only interested in invertible matrices, it still becomes necessary to consider noninvertible matrices. In the same way, if a connected group is the group of units of a connected algebraic monoid, then it is only natural to study the monoid and see if its study sheds some light on the structure of the group. A basic concept in $\Re_{n}(K)$ is that of rank $\rho$. The correct generalization of rank to an abstract monoid $S$ is given by the Green's relation $\mathscr{f}$ which is defined as: $a \mathscr{f} b$ if and only if $x a y=b, s b t=a$ for some $x, y, s, t \in S$. The rank ordering in $\mathfrak{K}_{n}(K)$ generalizes to a partial ordering of the $\mathcal{G}$-classes of $S$. A $\mathcal{G}$-class with an idempotent is called regular. Let $\mathcal{U}(S)$ denote the set of all regular $\mathcal{G}$-classes of $S$. It turns out that $(\mathcal{Q}(S), \leqslant)$ is a lattice if $S$ is a connected monoid. Let us now look at two basic connected monoids. Let $S_{1}=\mathfrak{K}_{n}(K), S_{2}=$ the monoid of all upper triangular $n \times n$ matrices over $K$. Let $G_{i}$ denote the group of units of $S_{i}(i=1,2) . G_{2}$ is

Received by the editors November 14, 1980 and, in revised form, May 5, 1981.

1980 Mathematics Subject Classification. Primary 20M10; Secondary 20 G99.

Key words and phrases. Connected monoids, solvable groups, $\mathscr{G}$-class, lattice, semilattice, idempotents.

${ }^{1}$ This research was partially supported by NSF Grant MCS 8025597. 
solvable while $G_{1}$ is as far from being solvable as possible. Now let us compare $\mathscr{Q}\left(S_{1}\right)$ and $\mathscr{Q}\left(S_{2}\right)$. $\mathcal{U}\left(S_{1}\right)$ is a linear chain while $\mathscr{U}\left(S_{2}\right)$ is a Boolean lattice. The basic conjecture in this direction is the following.

Conjecture. Let $S$ be a connected monoid with zero and group of units $G$. Then $G$ is solvable if and only if $\mathscr{U}(S)$ is a relatively complemented lattice. In this paper, we prove the conjecture when $|थ(S)| \leqslant 7$.

Let $S$ be a connected monoid with groups of units $G$. The connection between the solvability of $G$ and the semilattice decomposition of $S$ into archimedean semigroups was pointed out in [9]. This point is further elaborated in the present paper. If $J \in \mathcal{Q}(S)$, then we introduce two basic numbers $\theta(J)$ and $\delta(J)$ and study their properties. Crucial to this process is the theorem that for any idempotent $e$ of $S$, the centralizer of $e$ in $G$ is connected. If $S$ is a connected regular monoid, then the set $\{e \mid e \in E(S), e \geqslant f\}$ is finite for all $f \in E(S)$, if and only if $S$ is a Clifford semigroup. Connected monoids with central idempotents are also studied. A conjecture about their structure is forwarded. Let $S$ be a connected monoid. Then it is pointed out that the maximal connected submonoids of $S$ with central idempotents need not be conjugate. However special maximal connected submonoids with central idempotents are conjugate.

Let $S$ be a connected monoid with group of units $G$ and let $T$ be a maximal torus of $G$. The maximal semilattice image of $S$ is explicitly determined and is shown to be isomorphic to a subsemilattice of $(\mathcal{Q}(S), \wedge)$ and of $(E(\bar{T}), \cdot)$.

1. Preliminaries. Throughout this paper $Z^{+}, \mathbf{R}$ will denote the sets of positive integers and all reals, respectively. If $X$ is a set then $|X|$ denotes the cardinality of $X$. If $(P, \leqslant)$ is a partially ordered set, $\alpha, \beta \in P$, then $\beta$ covers $\alpha$ if $\beta>\alpha$ and there is no $\gamma \in P$ such that $\beta>\gamma>\alpha$. A linearly ordered subset of $P$ is a chain. The length of a chain $\left\{\alpha_{1}<\cdots<\alpha_{k}\right\}$ is $k-1$. A chain not contained in any other is a maximal chain. If $\xi \in P$, then $\xi$ is a minimal element of $P$ if there is no $\alpha \in P$ such that $\alpha<\xi$. $\xi$ is a minimum element of $P$ if $\xi \leqslant \alpha$ for all $\alpha \in P$. Let $(L, \vee, \wedge)$ be a lattice. Then $L$ is relatively complemented if for all $\alpha, \beta, \gamma \in L, \alpha>\beta>\gamma$ implies that there exists $\xi \in L$ such that $\beta \wedge \xi=\gamma$ and $\beta \vee \xi=\alpha$.

Let $S$ be a monoid with group of units $G$. If $X, Y \subseteq S$, then $X, Y$ are conjugate if $a^{-1} X a=Y$ for some $a \in G$. If $X \subseteq S$ and $Y \subseteq G$, then $N_{Y}(X)=\{y \mid y \in Y$, $\left.y^{-1} X y=X\right\}$ is the normalizer of $X$ in $Y$. If $X \subseteq S, Y \subseteq S$, then $C_{Y}(X)=\{y \mid y \in Y$, $x y=y x$ for all $x \in X\}$ is the centralizer of $X$ in $Y . C(S)=C_{S}(S)$ is the center of $S$. Let $S$ be a semigroup. If $a, b \in S$, then $a \mid b$ ( $a$ divides $b$ ) if $x a y=b$ for some $x, y \in S^{1} . S$ is archimedean if for all $a, b \in S, a \mid b^{i}$ for some $i \in Z^{+}$. Let $S_{\alpha}$ ( $\alpha \in \Omega$ ) denote a partition of $S$ into subsemigroups. Then $S$ is a semilattice (union) of $S_{\alpha}(\alpha \in \Omega)$ if for all $\alpha, \beta \in \Omega$, there exists $\gamma \in \Omega$ such that $S_{\alpha} S_{\beta} \cup S_{\beta} S_{\alpha} \subseteq S_{\gamma}$. By the author [4], $S$ is a semilattice of archimedean semigroups if and only if for all $a, b \in S, a \mid b$ implies $a^{2} \mid b^{i}$ for some $i \in Z^{+}$. A semigroup with no proper semilattice decomposition is said to be $\delta$-indecomposable. By Tamura $[\mathbf{1 0}, \mathbf{1 1}, \mathbf{1 2}]$ every semigroup is a semilattice of $\delta$-indecomposable semigroups. We let $E(S)$ denote the set of all idempotents of $S$. If $X \subseteq S$, then $E(X)=E(S) \cap X$. If $e, f \in E(S)$, then $e \geqslant f$ if $e f=f e=f$. We write $E(S)$ for the partially ordered set $(E(S), \leqslant) . \mathscr{G}, \Re, \mathcal{L}$, 
$\mathcal{H}, \mathcal{D}$ will denote the usual Green's relations on $S$. If $a \in S$, then $H_{a}, J_{a}$ will denote the $\mathcal{F}$-class and $\mathcal{G}$-class of $a$, respectively. We let $\mathcal{Q}(S)=\{J \mid J$ is a $\mathcal{G}$-class of $S$ and $E(J) \neq \varnothing\}$. If $J_{1}, J_{2} \in \mathcal{Q}(S)$, then $J_{1} \geqslant J_{2}$ if $a_{1} \mid a_{2}$ for some (all) $a_{i} \in J_{i}, i=1,2$. We write $थ(S)$ for the partially ordered set $(थ(S), \leqslant)$.

$K$ will denote a fixed algebraically closed field. If $n \in Z^{+}$, then $K^{n}=K \times \cdots \times K$ is the affine $n$-space and $\mathfrak{T}_{n}(K)$ the multiplicative monoid of all $n \times n$ matrices over $K$. If $X \subseteq K^{n}$, then $X$ is closed if $X$ is the set of zeros of some polynomials on $K^{n}$. If $X \subseteq K^{n}$, the $\bar{X}$ denotes the closure of $X$. By an algebraic semigroup we mean a closed subset of $K^{n}$ along with an associative operation which is a polynomial map. By a homomorphism between algebraic semigroups we mean a semigroup homomorphism which is also a morphism of varieties (= a polynomial map). By a connected semigroup we mean an algebraic semigroup such that the underlying closed set is irreducible (i.e., is not a union of two proper closed subsets). Let $S$ be an algebraic monoid with group of units $G$. Then $S$ is isomorphic to a closed submonoid of $\mathscr{K}_{n}(K)$ for some $n \in Z^{+}$. A power of each element of $S$ lies in a subgroup of $S$, $\mathcal{G}=\mathscr{Q}$, and $\mathcal{Q}(S)$ is finite. If $J \in \mathcal{Q}(S)$, then $J^{0}$ is completely 0 -simple. $G$ is a principal open subset of $S$ and can therefore be viewed as an algebraic group. $S \backslash G$ is a closed prime ideal of $S$. If $S_{1}$ is a closed submonoid of $S$, then $G_{1}=S_{1} \cap G$ is the group of units of $S_{1}$. The identity element 1 of $S$ lies in a unique irreducible component $S^{c}$ of $S$. Moreover $S^{c}$ is a closed connected submonoid of $S$ and $S^{c} \cap G=G^{c}$. If $S_{1}$ is a closed submonoid of $S$ then $S_{1}^{c} \cap G=\left(S_{1} \cap G\right)^{c}$. If $S$ is connected, then so is $G$, and in that case $\bar{G}=S$. If $e \in E(S)$, then $H_{e}$ is the group of units of $e S e$. So $H_{e}$ is an algebraic group. If $S$ is connected, then so is $H_{e}$ and $\bar{H}_{e}=e S e$.

We will need a number of basic results on (linear) algebraic groups. See [1, 3] for the extensive literature on algebraic groups. Let $G$ be a connected algebraic group and let $T$ be a maximal torus of $G$. We let $W(G)=N_{G}(T) / C_{G}(T)$ denote the Weyl group of $G$. Then [3, Proposition 24.1A, Corollary 25.2C], $W(G)$ is a finite group, and $G$ is solvable if and only if $|W(G)|=1$.

LEMMA 1.1. Let $S$ be an algebraic monoid, $S_{1}, S_{2}$ closed submonoids of $S$. Then $\left(S_{1}^{c} \cap S_{2}^{c}\right)^{c}=\left(S_{1}^{c} \cap S_{2}\right)^{c}=\left(S_{1} \cap S_{2}\right)^{c}$.

Proof. $S_{1}^{c} \cap S_{2}^{c} \subseteq S_{1}^{c} \cap S_{2} \subseteq S_{1} \cap S_{2}$. Hence $\left(S_{1}^{c} \cap S_{2}^{c}\right) \subseteq\left(S_{1}^{c} \cap S_{2}\right)^{c} \subseteq$ $\left(S_{1} \cap S_{2}\right)^{c} \subseteq S_{1}^{c} \cap S_{2}^{c}$. This yields the result.

LEMMA 1.2. Let $S$ be an algebraic monoid with group of units $G$. Then

(1) If $X, Y \subseteq S$, then $\overline{X Y}=\overline{\bar{X}} \bar{Y}$.

(2) If $X \subseteq S, a, b \in G$, then $\overline{a X b}=a \bar{X} b$.

(3) If $X \subseteq S, Y \subseteq G$, then $N_{Y}(X) \subseteq N_{Y}(\bar{X})$.

(4) If $X, Y \subseteq S$, then $C_{Y}(X)=C_{Y}(\bar{X})$.

Proof. (1) The set $\{x \mid x \in S, x Y \subseteq \overline{X Y}\}$ is closed and contains $X$. Hence $\bar{X} Y$ $\subseteq \overline{X Y}$. Similarly $\bar{X} \bar{Y} \subseteq \overline{X Y}$. Since $X Y \subseteq \bar{X} \bar{Y}, \overline{X Y}=\overline{\bar{X}} \bar{Y}$.

(2) Since $a, b \in G, a \bar{X} b$ is closed. Since $a X b \subseteq a \bar{X} b, \overline{a X b} \subseteq a \bar{X} b$. Similarly, since $X \subseteq a^{-1} \overline{a X b} b^{-1}$, we see that $\bar{X} \subseteq a^{-1} \overline{a X b} b^{-1}$. 
(3) Let $a \in N_{Y}(X)$. Then $a^{-1} X a=X$. By (2), $a^{-1} \bar{X} a=\bar{X}$.

(4) Let $y \in C_{Y}(X)$. Then $C_{S}(y)$ is a closed set containing $X$. Hence $\bar{X} \subseteq C_{S}(y)$. So $y \in C_{Y}(\bar{X})$.

LEMMA 1.3. Let $S$ be a connected monoid with group of units $G$. Let $T$ be a maximal torus of $G, e \in E(\bar{T})$. Let $S_{1}=\{a \mid a \in S, a e=e a=e\}, T_{1}=\{a \mid a \in T$, ae $=e a$ $=e\}, S_{2}=S_{1}^{c}, T_{2}=T_{1}^{c}, G_{2}$ the group of units of $S_{2}$. Then

(1) for all $f \in E(S), f \geqslant e$ implies $f \in S_{2}$;

(2) $T_{2}$ is a maximal torus of $G_{2}$;

(3) if $e$ is a minimal idempotent of $S$, then $E(\bar{T})=E\left(\bar{T}_{2}\right)$.

Proof. (1) By [7, Lemma 1.1], there exists a closed connected submonoid of $S^{\prime}$ of $S$ such that $f$ is the zero of $S^{\prime}$. Then $S^{\prime} \subseteq S_{1}$. Hence $S^{\prime} \subseteq S_{1}^{c}=S_{2}$. So $f \in S_{2}$.

(2) Let $\Gamma=\left\{1>e_{1}>\cdots>e_{k}=e>e_{k+1}>\cdots>e_{m}\right\}$ be a maximal chain in $E(\bar{T})$. Then by [9, Lemma 17], $\Gamma$ is a maximal chain in $E(S)$. Let $\Gamma_{0}=\left\{1>e_{1}>\right.$ $\left.\cdots>e_{k}=e\right\}$. Then by (1) (applied to $\left.\bar{T}\right), \Gamma_{0} \subseteq E\left(\bar{T}_{2}\right)$. Clearly $\Gamma_{0}$ is a maximal chain in $E\left(S_{2}\right)$. Since $e$ is the zero of $\bar{T}_{2}$, we see by [6, Theorem 3.17] that $\operatorname{dim} T_{2}=k$. Let $T_{2} \subseteq T_{3}$ where $T_{3}$ is a maximal torus of $G_{2}$. Then clearly $\Gamma_{0}$ is a maximal chain in $E\left(\bar{T}_{3}\right)$ and $e$ is the zero of $\bar{T}_{3}$. So again by [6, Theorem 3.17], $\operatorname{dim} T_{3}=k$. So $T_{2}=T_{3}$.

(3) If $e$ is a minimal idempotent of $S$, then clearly $e$ is the minimum idempotent of $\bar{T}$ and so by (1) (applied to $\bar{T}), E(\bar{T}) \subseteq E\left(\bar{T}_{2}\right)$.

LEMMA 1.4. Let $S$ be a connected monoid with group of units $G$. Let $T$ be a maximal torus of $G, e \in E(\bar{T})$. Then $e T$ is a maximal torus of $H_{e}$.

Proof. Clearly $T \subseteq C_{G}(e)^{c}$. Consider the homomorphism, $\phi: C_{G}(e)^{c} \rightarrow H_{e}$ given by $\phi(a)=e a$. By [8, Theorem 4], $\phi$ is surjective. Hence by [3, Corollary 21.3C], $\phi(T)=e T$ is a maximal torus of $H_{e}$.

LEMMA 1.5. Let $S$ be a connected monoid with group of units $G$. Let $T$ be a maximal torus of $G$. Let $e, f \in E(S)$ such that $e \mid f$. Then

(1) if $e \in E(\bar{T})$, there exists $f^{\prime} \in E(\bar{T})$ such that $e \geqslant f^{\prime}$ and $f \mathscr{f} f^{\prime}$;

(2) if $f \in E(\bar{T})$, there exists $e^{\prime} \in E(\bar{T})$ such that $e^{\prime} \geqslant f$ and $e^{\prime} f e$.

Proof. (1) By [6, Lemma 1.3], there exists $f_{1} \in E(S)$ such that $f \mathscr{f} f_{1}$ and $e \geqslant f_{1}$. By Lemma 1.4, eT is a maximal torus of $H_{e}$. Since $H_{e}$ is the group of units of $e S e$ and since $f_{1} \in e S e$, we see [7, Theorem 1.8] that there exists $f^{\prime} \in E(\overline{e T})$ such that $f_{1} g f^{\prime}$. So $f^{\prime} \in E(\bar{T}), e \geqslant f^{\prime}$.

(2) By [8, Theorem 10], there exists $e_{1} \in E(S)$ such that $e g e_{1}$ and $e_{1} \geqslant f_{1}$. Let $S_{1}=\{a \mid a \in S$, af $=f a=f\}, T_{1}=\{a \mid a \in T$, af $=f\}, S_{2}=S_{1}^{c}, T_{2}=T_{1}^{c}, G_{2}$ be the group of units of $S_{2}$. Then by Lemma $1.3, e_{1}, f \in S_{2}$ and $T_{2}$ is a maximal torus of $G_{2}$. So by [7, Theorem 1.8] there exists $e^{\prime} \in E\left(\bar{T}_{2}\right)$ such that $e_{1} g e^{\prime}$. Then $e^{\prime} \geqslant f$, $e^{\prime} \in E(\bar{T})$.

LEMMA 1.6. Let $S$ be a connected monoid with group of units $G$. Let $T$ be a maximal torus of $G$ and let $e, f \in E(\bar{T}), e>f$. Then the following conditions are equivalent:

(1) $e$ covers $f$ in $E(S)$.

(2) e covers $f$ in $E(\bar{T})$.

(3) $J_{e}$ covers $J_{f}$ in $थ(S)$. 
Proof. That (1) $\Rightarrow(2)$ is obvious. So assume (2). Suppose $J_{e}$ does not cover $J_{f}$. Then there exists $e_{1} \in E(S)$ such that $e_{1} \notin J_{e}, e_{1} \notin J_{f}$ and $e\left|e_{1}\right| f$. By [6, Lemma 1.3], there exists $e_{2} \in E(S)$ such that $e>e_{2}, e_{2} g e_{1}$. So $e_{2}, f \in e S e$. By Lemma 1.4, $e T$ is a maximal torus of $H_{e}$. By [6, Lemma 1.7], $e_{2} \mid f$ in $e S e$. So by Lemma 1.5, there exists $e_{3} \in E(\overline{e T})$ such that $e_{3} g e_{2}$ and $e_{3}>f$. Then $e>e_{3}>f, e_{3} \in E(\bar{T})$. This contradiction shows that (2) $\Rightarrow(3)$. Next assume (3). Suppose $e>e_{1}>f$ for some $e_{1} \in E(S)$. Then $J_{e}>J_{e_{1}}>J_{f}$, a contradiction. Hence (3) $\Rightarrow(1)$.

LEMMA 1.7. Let $S$ be a connected monoid with group of units $G$ and let $T$ be a maximal torus of $G$.

(1) If $J \in \mathcal{Q}(S), e \in J \cap E(\bar{T})$, then $J \cap E(\bar{T})=\left\{x^{-1}\right.$ ex $\left.\mid x \in N_{G}(T)\right\}$.

(2) If $J_{1}, J_{2} \in \mathcal{Q}(S)$, then $J_{1}>J_{2}$ if and only if there exist $e_{i} \in J_{i} \cap E(\bar{T}), i=1,2$, such that $e_{1}>e_{2}$.

Proof. (1) Let $f \in E(\bar{T}) \cap J$. Then by [8, Theorem 9], $x^{-1} e x=f$ for some $x \in G$. Since $T \subseteq C_{G}(f), x T x^{-1} \subseteq C_{G}(e)$. So $T, x T x^{-1}$ are maximal tori of $C_{G}(e)^{c}$. Hence there exists $y \in C_{G}(e)^{c}$ such that $y^{-1} T y=x T x^{-1}$. Then $x^{-1} y^{-1} T y x=T$. So $y x \in$ $N_{G}(T)$. Since $y \in C_{G}(e),(y x)^{-1} e y x=x^{-1} e x=f$. This proves (1).

(2) By [7, Theorem 1.8], there exists $e_{1} \in J_{1} \cap E(\bar{T})$. If $J_{1}>J_{2}$, then by Lemma 1.5 , there exists $e_{2} \in J_{2} \cap E(\bar{T})$ such that $e_{1}>e_{2}$. The converse is trivial.

LeMma 1.8. let $S$ be a connected monoid, $e \in E(S)$. Then $\mathscr{V}=\{J \mid J \in \mathcal{Q}(S)$, $\left.J \leqslant J_{e}\right\}$ is a sublattice of $\mathcal{U}(S)$ and $\mathscr{U}(e S e)=\{J \cap e S e \mid J \in \mathscr{V}\} \cong \mathscr{V}$.

Proof. Let $J \in \mathcal{T}, f \in E(J)$. Then $e \mid f$. By [6, Lemma 1.3], $e \geqslant f^{\prime}$ for some $f^{\prime} \in E(J)$. So $f^{\prime} \in J \cap e S e$. It follows by [6, Lemma 1.7] that $J \cap e S e \in \mathcal{Q}(e S e)$. The rest follows from [6, Lemma 1.7].

Corollary 1.9. Let $S$ be a connected monoid, $e \in E(S)$. If $\mathcal{Q}(S)$ is a relatively complemented lattice, then so is $\mathcal{Q}(e S e)$.

LEMMA 1.10. Let $S$ be a connected monoid, $e \in E(S)$. Suppose eSe is a semilattice of archimedean semigroups. Let $f_{1}, f_{2} \in E(S)$ such that $e \geqslant f_{1}, e \geqslant f_{2}, f_{1} \mathscr{f} f_{2}$ and $f_{1} f_{2}=f_{2} f_{1}$. Then $f_{1}=f_{2}$.

Proof. Let $f_{1}, f_{2} \in J \in \mathcal{U}(S)$. Then by Lemma 1.8, $f_{1}, f_{2} \in J \cap e S e \in \mathcal{Q}(e S e)$. By [9, Proposition 21] $J \cap e S e$ is completely simple. Since $f_{1} f_{2}=f_{2} f_{1}$ we see that $f_{1}=f_{2}$.

LEMMA 1.11. Let $S$ be a closed connected subsemigroup of $\mathfrak{M}_{n}(K)$ and let $J \in \mathcal{Q}(S)$. Suppose that for all $e, f \in E(J)$, any eigenvalue of ef is either 0 or 1 . Then $J^{2} \subseteq J$.

Proof. By the proof of [9, Proposition 23], $E(J)^{2} \subseteq E(J)$. Since $J^{0}$ is completely 0 -simple, it follows easily that $J^{2} \subseteq J$.

LeMma 1.12. Let $S$ be a connected semigroup, $J \in \mathcal{Q}(S)$. If $x, y \in J$, then there exist $e_{1}, e_{2} \in E(J)$ such that $x \Re e_{1} \& e_{2} \Re y$.

Proof. $J^{0}$ is completely 0 -simple, and hence admits a Rees representation $J^{0}=(\Gamma \times G \times \Lambda) \cup\{0\}$ with sandwich map $P: \Lambda \times \Gamma \rightarrow G^{0}$ where $G$ is a group. 
Multiplication in $J^{0}$ is given by

$$
(\alpha, a, \beta)(\gamma, b, \delta)=\left\{\begin{array}{l}
(\alpha, a P(\beta, \gamma) b, \delta) \quad \text { if } P(\beta, \gamma) \neq 0, \\
0 \text { if } P(\beta, \gamma)=0 .
\end{array}\right.
$$

Let $x=\left(\alpha_{1}, a_{1}, \beta_{1}\right), y=\left(\alpha_{2}, a_{2}, \beta_{2}\right)$. By [5, Theorem 2.16] there exists $\mu \in \Lambda$ such that $P\left(\mu, \alpha_{1}\right) \neq 0, P\left(\mu, \alpha_{2}\right) \neq 0$. Let $e_{1}=\left(\alpha_{1}, P\left(\mu, \alpha_{1}\right)^{-1}, \mu\right), e_{2}=$ $\left(\alpha_{2}, P\left(\mu, \alpha_{2}\right)^{-1}, \mu\right)$. Then $e_{1}, e_{2} \in E(J)$ and $x \Re e_{1} \mathcal{L} e_{2} \Re y$.

LEMMA 1.13. Let $S$ be a commutative connected monoid, $e \in E(S)$. If $\Omega$ is a finite group of automorphisms of $S$ having $e$ as a common fixed point, then there exists a closed connected submonoid $S_{1}$ of $S$ such that $e \in S_{1}$ and $\phi(a)=a$ for all $\phi \in \Omega$, $a \in S_{1}$.

Proof. Let $\Omega=\left\{\phi_{1}, \ldots, \phi_{k}\right\}$ and define $\psi: S \rightarrow S$ as $\psi(x)=\phi_{1}(x) \cdots \phi_{k}(x)$. Since $S$ is commutative, $\psi$ is a homomorphism. Clearly $\psi(1)=1$ and $\psi(e)=e$. Let $\phi \in \Omega$. Then since $\phi \Omega=\Omega, \phi \psi(x)=\psi(x)$ for all $x \in S$. Let $S_{1}=\overline{\psi(S)}$. Then $S_{1}$ is a closed connected submonoid of $S$ and $e \in S_{1}$. Clearly $\phi(a)=a$ for all $\phi \in \Omega$ and $a \in S_{1}$.

Proposition 1.14. Let $S$ be a connected monoid with group of units $G$ and let $T$ be $a$ maximal torus of $G$. Let $\mathcal{X}=\{J \mid J \in \mathcal{Q}(S), G$ covers $J\}, \mathcal{Y}=\left\{J \mid J \in \mathcal{Q}(S), J_{1}\right.$ covers $J$ for some $\left.J_{1} \in \mathcal{X}\right\}, X=\{e \mid e \in E(\bar{T}), 1$ covers $e\}, Y=\{f \mid f \in E(\bar{T}), e$ covers for some $e \in X\}$. Then

(1) $X=\cup_{J \in \mathscr{X}} E(\bar{T}) \cap J$.

(2) $Y=\cup_{J \in \mathcal{Y}} E(\bar{T}) \cap J$.

(3) If $J_{1} \in \mathcal{Q}(S), J_{2} \in \mathcal{Y}$ and if $G>J_{1}>J_{2}$, then $J_{1} \in \mathcal{X}$ and $J_{1}$ covers $J_{2}$.

(4) If $e \in E(\bar{T}), f \in Y$, and if $1>e>f$, then $e$ covers $f$ and $e \in X$.

(5) If $J^{\prime} \in \mathcal{Y}$, then $\left|\left\{J \mid J \in \mathcal{X}, J>J^{\prime}\right\}\right| \leqslant 2$.

(6) If $f \in Y$, then $|\{e \mid e \in X, e>f\}| \leqslant 2$.

(7) If $\mathcal{U}(S)$ is relatively complemented, if $f \in Y, e_{1}, e_{2} \in X, e_{1}>f, e_{2}>f$, and if $e_{1} g e_{2}$, then $e_{1}=e_{2}$.

Proof. (1), (2) follow from Lemmas 1.5 and 1.6. Next let $J_{2} \in \mathcal{Y}, J_{1} \in \mathcal{Q}(S)$ such that $G>J_{1}>J_{2}$. There exists $J \in \mathcal{X}$ such that $J$ covers $J_{1}$. So we have a maximal chain $\Gamma=\left\{G>J>J_{2}>J_{3}>\cdots>J_{t}\right\}$ in $\mathcal{U}(S)$. Then $\Lambda=\left\{G>J_{1}>J_{2}>J_{3}>\right.$ $\left.\cdots>J_{t}\right\}$ is a chain in $\mathcal{Q}(S)$. Since by [7, Theorem 1.9] all maximal chains in $\mathcal{Q}(S)$ have the same length, $\Lambda$ is also a maximal chain in $\mathcal{Q}(S)$. So $G$ covers $J_{1}$ and $J_{1}$ covers $J_{2}$. This proves (3). Now (4) follows from Lemma 1.6.

We now prove (5). Let $J_{1}, J_{2}, J_{3}$ be distinct elements of $\mathcal{X}, J^{\prime} \in \mathcal{Y}$ such that $J_{i}>J^{\prime}, i=1,2,3$. Let $f \in J^{\prime} \cap E(\bar{T})$. By Lemma 1.5, there exists $e_{i} \in J_{i} \cap E(\bar{T})$, $i=1,2,3$, such that $e_{i}>f, i=1,2,3$. Then by (1), (2), $e_{1}, e_{2}, e_{3} \in X$ and $f \in Y$. Let $T_{1}=\{a \mid a \in T, a f=f\}, T_{2}=T_{1}^{c}$. Then by Lemma 1.3, $e_{1}, e_{2}, e_{3}, f \in \bar{T}_{2}$. Clearly $f$ is the zero of $\bar{T}_{2}$. By (4), $\left\{1>e_{1}>f\right\}$ is a maximal chain in $E\left(\bar{T}_{2}\right)$. By [6, Theorem 3.17], $\operatorname{dim} \bar{T}_{2}=2$. So by [6, Theorem 3.18], $\left|E\left(\bar{T}_{2}\right)\right| \leqslant 4$. However, $1, e_{1}, e_{2}, e_{3}, f$ are distinct elements of $E\left(\bar{T}_{2}\right)$. This contradiction proves (5). We now obtain (6) by applying (5) to $\bar{T}$. 
Next we prove (7). Suppose $\mathscr{Q}(S)$ is relatively complemented, $e_{1}, e_{2} \in X$, $e_{1} g e_{2}, e_{1} \neq e_{2}, e_{1}>f, e_{2}>f$. Let $J_{1}$ denote the $\mathcal{G}$-class of $e_{1}, J^{\prime}$ the $\mathcal{G}$-class of $f$. Then $J_{1} \wedge J=J^{\prime}, J_{1} \vee J=G$ for some $J \in \mathcal{U}(S)$. By (1), (2), (3), $J_{1}, J \in \mathcal{X}$ and $J^{\prime} \in \mathcal{Y}$. By Lemma 1.5, there exists $e_{3} \in J \cap E(\bar{T})$ such that $e_{3}>f$. By (1), $e_{3} \in X$. So $e_{i}>f, i=1,2,3$. This contradicts (6), proving (7).

LEMMA 1.15. Let $S$ be a connected monoid such that the group of units $T$ of $S$ is a torus. Let $X=\{e \mid e \in E(S), 1$ covers $e\}, Y=\{f \mid f \in E(S)$, e covers $f$ for some $e \in X\}$. Then for any $e, e^{\prime} \in X$ with $e \neq e^{\prime}$, there exist distinct $f_{1}, \ldots, f_{k} \in Y$ and distinct $e_{1}, \ldots, e_{k+1} \in X$ such that $e=e_{1}, e^{\prime}=e_{k+1}$ and $e_{i}>f_{i}, e_{i+1}>f_{i}, i=1, \ldots, k$.

Proof. By Lemma 1.3, we can assume that $S$ has a zero. If $P$ is a polytope in $\mathbf{R}^{n}$, then let $\mathscr{F}(P)$ denote the lattice of all faces of $P$ (see [2]). By [7, Theorem 3.6], $E(S)$ is isomorphic to $\mathscr{F}(P)$ for a polytope $P$ in some $\mathbf{R}^{n}$. Let $P^{x}$ denote the dual polytope of $P\left[2, \S 3.4\right.$, p. 46]. Then $\mathscr{F}(P)$ is anti-isomorphic to $\mathscr{F}\left(P^{*}\right)$. Let $\phi: E(S) \rightarrow \mathscr{F}\left(P^{*}\right)$ denote the corresponding map. Then $\phi(X)$ consists of all vertices of $P^{*}$ and $\phi(Y)$ consists of all edges of $P$. By a result of Balinski (see [2, $\$ 11.3$, Theorem 2]) there exist vertices $u_{1}, \ldots, u_{k+1}$ and edges $v_{1}, \ldots, v_{k}$ such that $\phi(e)=u_{1}, \phi\left(e^{\prime}\right)=u_{k+1}$ and $u_{i}, u_{i+1}$ are the vertices of $v_{i}, i=1, \ldots, k$. Let $e_{i}=\phi^{-1}\left(u_{i}\right), i=1, \ldots, k+1, f_{i}=$ $\phi^{-1}\left(v_{i}\right), i=1, \ldots, k$. Then $e_{i}>f_{i}, e_{i+1}>f_{i}, i=1, \ldots, k$. If we choose $k$ to be minimal, then clearly, $e_{1}, \ldots, e_{k+1}$ and $f_{1}, \ldots, f_{k}$ will be distinct.

LEMMA 1.16. Let $G$ be a connected group, $H$ a closed connected normal subgroup of $G, B_{0}$ a Borel subgroup of $G$ such that $B_{0} H=G$. Then $|W(G)|=|W(H)|$.

Proof. Since $H \triangleleft G$ and since all Borel subgroups of $G$ are conjugate, $B H=G$ for all Borel subgroups $B$ of $G$. Let $U$ be a Borel subgroup of $H$. Then $U \subseteq V$ for some Borel subgroup $V$ of $G$. Then $U \subseteq V \cap H$ and $V \cap H$ is solvable. By [3, Corollary 23.1A] $V \cap H=U$ is a Borel subgroup of $H$. Since $H \triangleleft G, B \cap H$ is a Borel subgroup of $H$ for any Borel subgroup $B$ of $G$. Let $T_{1}$ be a maximal torus of $H$ and let $\mathbb{Q}=\left\{B \mid B\right.$ is a Borel subgroup of $\left.G, T_{1} \subseteq B\right\}, \mathbb{Q}_{1}=\{B \cap H \mid B \in \mathbb{Q}\}$. Then by [3, Proposition 24.1A], $\left|\mathbb{Q}_{1}\right|=|W(H)|$. Let $B_{1}, B_{2} \in \mathbb{Q}$ such that $B_{1} \cap H=B_{2}$ $\cap H$. Now $x^{-1} B_{1} x=B_{2}$ for some $x \in G=B_{1} H$. So $x=b h$ for some $b \in B_{1}$, $h \in H$. So $h^{-1} B_{1} h=B_{2}$. Thus $h^{-1}\left[B_{1} \cap H\right] h=B_{2} \cap H=B_{1} \cap H$. So $h \in$ $N_{H}\left(B_{1} \cap H\right)$. By [3, Theorem 23.1], $N_{H}\left(B_{1} \cap H\right)=B_{1} \cap H$. Thus $h \in B_{1} \cap H \subseteq$ $B_{1}$, and $B_{1}=B_{2}$. It follows that $|Q|=\left|Q_{1}\right|<\infty$. Hence $T_{1}$ is a regular torus of $G$ (see [3, Chapter IX]). Hence [3, Proposition 24.2], $|\mathscr{Q}|=|W(G)|$.

Corollary 1.17. Let $G$ be a connected group, $H$ a closed normal subgroup of $G, B$ a Borel subgroup of $G$ such that $B H=G$. Then $|W(G)|=\left|W\left(H^{c}\right)\right|$.

Proof. Since $H \triangleleft G, H^{c} \triangleleft G$. So $B H^{c}$ is a subgroup of $G$. Since $B H^{c}$ is the image of the product map from $B \times H^{c}$ into $G, B H^{c}$ is a constructible subgroup of $G$. Hence [3, Proposition 7.4A], $B H^{c}$ is a closed subgroup of $G$. By [3, Proposition 7.3], $H=H^{c} h_{1} \cup \cdots \cup H^{c} h_{k}$ for some $h_{1}, \ldots, h_{k} \in H$. Hence $G=B H=B H^{c} h_{1}$ $\cup \cdots \cup B H^{c} h_{k}$. Since $G$ is connected, $G=B H^{c} h_{i}$ for some $i$. So $h_{i} \in B H^{c}$ and $G=B H^{c}$. We are now done by Lemma 1.16 . 
COROLlaRY 1.18. Let $\phi: G \rightarrow G^{\prime}$ be a surjective homomorphism of connected groups such that $G^{\prime}$ is solvable and let $H$ denote the kernel of $\phi$. Then $|W(G)|=\left|W\left(H^{c}\right)\right|$.

Proof. Let $B$ be a Borel subgroup of $G$. Then by [3, Corollary 21.3C], $\phi(B)=G^{\prime}$. So $G=B H$. We are done by Corollary 1.17 .

LEMMA 1.19. Let $\phi: G \rightarrow G^{\prime}$ be a surjective homomorphism of connected groups and let $H$ denote the kernel of $\phi$. Then $|W(G)|=\left|W\left(H^{c}\right)\right|\left|W\left(G^{\prime}\right)\right|$.

Proof. Let $T$ be a maximal torus of $G$. Then by [3, Corollary 21.3C], $T^{\prime}=\phi(T)$ is a maximal torus of $G^{\prime}$. Let $\mathcal{Q}=\{B \mid B$ is a Borel subgroup of $G, T \subseteq B\}, \mathbb{Q}^{\prime}=\left\{B^{\prime} \mid B^{\prime}\right.$ is a Borel subgroup of $\left.G^{\prime}, T^{\prime} \subseteq B^{\prime}\right\}$. By [3, Chapter IX], $|\mathbb{Q}|=|W(G)|,\left|\mathbb{Q}^{\prime}\right|=$ $\left|W\left(G^{\prime}\right)\right|$. If $B \in \mathbb{Q}$, then by [3, Corollary $\left.21.3 \mathrm{C}\right], \phi(B) \in \mathbb{Q}^{\prime}$. If $B^{\prime} \in \mathbb{Q}^{\prime}$, then let $\mathbb{Q}_{B^{\prime}}=\left\{B \mid B \in \mathbb{Q}, \phi(B)=B^{\prime}\right\}$. Then clearly $|\mathbb{Q}|=\Sigma_{B^{\prime} \in \mathbb{Q}^{\prime}}\left|\mathbb{Q}_{B^{\prime}}\right|$. Let $B^{\prime} \in \mathbb{Q}^{\prime}$. It suffices to show that $\left|W\left(H^{c}\right)\right|=\left|\mathbb{Q}_{B^{\prime}}\right|$. Let $G_{1}=\phi^{-1}\left(B^{\prime}\right)^{c}$. Then $T \subseteq G_{1}$. By [3, Corollary 21.3C], $\phi: G_{1} \rightarrow B^{\prime}$ is surjective. Let $H_{1}$ denote the kernel of this map. Then since $H^{c} \subseteq G_{1}, H^{c}=H_{1}^{c}$. Let $\mathcal{C}=\left\{B \mid B\right.$ is a Borel subgroup of $\left.G_{1}, T \subseteq B\right\}$. Let $B \in \mathcal{C}$. By [3, Corollary 21.3C], $\phi(B)=B^{\prime}$. Let $B \subseteq B_{1}, B_{1}$ a Borel subgroup of $G$. Then $B^{\prime} \subseteq \phi\left(B_{1}\right)$ and $\phi\left(B_{1}\right)$ is a Borel subgroup of $G^{\prime}$. Thus $B^{\prime}=\phi\left(B_{1}\right)$. Hence $B_{1} \subseteq G_{1}$ and $B=B_{1} \in \mathbb{Q}_{B^{\prime}}$. So $\mathcal{C}=\mathscr{Q}_{B^{\prime}}$. Thus $\left|\mathcal{Q}_{B^{\prime}}\right|=\left|W\left(G_{1}\right)\right|$. By Lemma 1.18, $\left|W\left(G_{1}\right)\right|=\left|W\left(H^{c}\right)\right|$. This proves the lemma.

COROLLARY 1.20. Let $S$ be a connected monoid with group of units $G, e \in E(C(S))$. Let $G_{1}=\{a \mid a \in G, a e=e\}$. Then $|W(G)|=\left|W\left(G_{1}^{c}\right)\right|\left|W\left(H_{e}\right)\right|$.

Proof. The homomorphism $\phi: G \rightarrow H_{e}$ is given by $\phi(x)=e x$ is surjective by [8, Lemma 3]. Clearly $G_{1}$ is the kernel of $\phi$. We are done by Lemma 1.19.

LEMMA 1.21. Let $S$ be an S-indecomposable semigroup, $J \in \mathcal{Q}(S)$. Then $J^{2} \subseteq J$ if and only if $J$ is the kernel of $S$.

Proof. By Tamura [10], $S^{1} J S^{1}$, being an ideal of $S$, is $\mathcal{S}$-indecomposable. If $J$ is not the kernel of $S$, then $\left\{J, S^{1} J S^{1} \backslash J\right\}$ is a proper semilattice decomposition of $S^{1} J S^{1}$, a contradiction.

2. Main section. Fundamental in the theory of connected algebraic monoids is the following theorem (see [7, Theorems 1.8, 3.6, Corollary 1.6]; [6, Theorem 3.20]).

THEOREM 2.1 [7]. Let $S$ be a connected monoid with group of units $G$ and let $T$ be a maximal torus of $G$. Then

(1) $\mathcal{Q}(S)$ is a finite lattice.

(2) $E(\bar{T})$ is a finite, relatively complemented lattice (in fact the lattice of all faces of a rational polytope in some $\mathbf{R}^{n}$ ).

(3) Any maximal chain in $\mathcal{Q}(S)$ has the same length as any maximal chain in $E(\bar{T})$ or $E(S)$.

(4) $E(S)=\cup_{x \in G} x^{-1} E(\bar{T}) x$.

See [8, Theorem 3] for the following theorem. 
THEOREM 2.2 [8]. Let $S$ be a connected monoid with group of units $G$. If $J \in \mathcal{Q}(S)$, and if $a, b \in J$, then $x a y=b$ for some $x, y \in G$. If $e, f \in E(J)$, then $x^{-1} e x=f$ for some $x \in G$.

A special case of the following basic result was proved in [9, Theorem 14].

THEOREM 2.3. Let $S$ be a connected monoid with group of units $G$. Then for any $e \in E(S), C_{G}(e)$ is a connected group.

Proof. By Theorem 2.1, $e \in \bar{T}$ for some maximal torus $T$ of $G$. First let $u \in C_{G}(e) \cap N_{G}(T)$. Since $N_{G}(T) / C_{G}(T)$ is a finite group [3, Chapter IX], $u^{i} \in$ $C_{G}(T)$ for some $i \in Z^{+}$. Consider the automorphism $\phi: \bar{T} \rightarrow \bar{T}$ given by $\phi(x)=$ $u^{-1} x u$. Since $u^{i} \in C_{G}(T)=C_{G}(\bar{T}), \phi^{i}$ is the identity map. Since $u \in C_{G}(e), \phi(e)=e$. By Lemma 1.13 there exists a subtorus $T_{1}$ of $T$ such that $e \in \bar{T}_{1}$ and $\phi(a)=a$ for $a \in T_{1}$. So $u \in C_{G}\left(T_{1}\right)$. By [3, Theorem 22.3], $C_{G}\left(T_{1}\right)$ is connected. On the other hand, since $e \in \bar{T}_{1}, C_{G}\left(T_{1}\right)=C_{G}\left(\bar{T}_{1}\right) \subseteq C_{G}(e)$. So $C_{G}\left(T_{1}\right) \subseteq C_{G}(e)^{c}$. Therefore,

$$
C_{G}(e) \cap N_{G}(T) \subseteq C_{G}(e)^{c} .
$$

Now let $a \in C_{G}(e)$. Then $T, a^{-1} T a$ are maximal tori of $C_{G}(e)^{c}$. So $b^{-1} a^{-1} T a b=T$ for some $b \in C_{G}(e)^{c}$. Thus $a b \in C_{G}(e) \cap N_{G}(T) \subseteq C_{G}(e)^{c}$. Since $b \in C_{G}(e)^{c}, a \in$ $C_{G}(e)^{c}$.

DEFINITION. Let $S$ be a connected monoid with group of units $G$ and let $T$ be a maximal torus of $G$.

(1) $\alpha(S)=|E(\bar{T})|$.

(2) $\beta(S)=\mid$ U $(S) \mid$.

(3) If $W$ is the Weyl group of $G$, then $\gamma(S)=|W|$.

(4) If $J \in \mathcal{Q}(S)$ and if $e \in E(J)$, then $\theta(e)=\theta(J)=|J \cap E(\bar{T})|$ and $\delta(e)=$ $\delta(J)=\gamma\left(C_{G}(e)\right)$.

We write $\theta_{S}, \delta_{S}$ if we want to specify $S$.

REMARK 2.4. Since all maximal tori in $G$ are conjugate, since all idempotents of $J$ are conjugate and since $x^{-1} J x=J$ for all $x \in G$, we see that the above definitions are independent of the choice of $T$ and the particular idempotent $e$ in $J$.

REMARK 2.5. If $S$ is a connected monoid and if $S_{1}$ is a closed connected submonoid of $S$, then $\alpha\left(S_{1}\right) \leqslant \alpha(S)$. However, it can very well happen that $\beta\left(S_{1}\right)$ is larger than $\beta(S)$. If $e \in E\left(S_{1}\right)$, then $\theta_{S_{1}}(e) \leqslant \theta_{S}(e)$.

THEOREM 2.6. Let $S$ be a connected monoid. Then

(1) For all $J \in \mathcal{U}(S), \gamma(S)=\theta(J) \delta(J)$. In particular, $1 \leqslant \theta(J) \leqslant \gamma(S)$.

(2) $\alpha(S)=\sum_{J \in \mathcal{Q}(S)} \theta(J)$.

(3) $\beta(S) \leqslant \alpha(S) \leqslant \beta(S) \gamma(S)$.

(4) If $J_{1}, J_{2} \in \mathcal{Q}_{S}$, then $\theta\left(J_{1} \wedge J_{2}\right) \leqslant \theta\left(J_{1}\right) \theta\left(J_{2}\right)$.

Proof. Let $J \in \mathcal{Q}(S), T$ a maximal torus of $G$, the group of units of $S$. Let $A=E(\bar{T}) \cap J$. Then $\theta(J)=|A|$. Let $e \in A$. If $x \in N_{G}(T)$ and $f \in A$, then let $f^{x}=x^{-1} f x \in A$. Clearly, $\left(f^{x}\right)^{y}=f^{x y}$ for all $x, y \in N_{G}(T)$. So the group $N_{G}(T)$ acts on $A$. By Lemma 1.7, $N_{G}(T)$ acts transitively on $A$. The stabilizer of $e$ is $V=C_{G}(e)$ $\cap N_{G}(T)$. Thus $\theta(J)=|A|=\left|N_{G}(T) / V\right|$. Clearly $T \subseteq C_{G}(T) \subseteq V$. So 


$$
\left|N_{G}(T) / V\right|\left|V / C_{G}(T)\right|=\left|N_{G}(T) / C_{G}(T)\right|
$$

Now $V$ is the normalizer of $T$ in $C_{G}(e)$ and $C_{G}(T)$ is the centralizer of $T$ in $C_{G}(e)$. So $\delta(e)=\left|V / C_{G}(T)\right|$. Clearly $\gamma(S)=\left|N_{G}(T) / C_{G}(T)\right|$. This proves (1). Clearly $\alpha(S)$ $=|E(\bar{T})|=\Sigma_{J \in \mathcal{Q}(S)}|J \cap E(\bar{T})|$. This yields (2). Now (3) follows from (1) and (2). Finally we prove (4). Let $h \in\left(J_{1} \wedge J_{2}\right) \cap E(\bar{T})$. Then by Lemma 1.5, there exists $e_{i} \in J_{i} \cap E(\bar{T})$ such that $e_{i} \geqslant h, i=1,2$. So $e_{1} e_{2} \geqslant h$. By Lemma 1.7, $e_{1} e_{2}=h$. So $\left(J_{1} \wedge J_{2}\right) \cap E(\bar{T}) \subseteq\left(J_{1} \cap E(\bar{T})\right)\left(J_{2} \cap E(\bar{T})\right)$. Hence $\theta\left(J_{1} \wedge J_{2}\right) \leqslant \theta\left(J_{1}\right) \theta\left(J_{2}\right)$.

COROLlary 2.7. Let $S$ be a connected monoid with group of units $G$ and let $T$ be $a$ maximal torus of $G$. Then the following conditions are equivalent.

(1) $\alpha(S)=\beta(S)$.

(2) $\theta(J)=1$ for all $J \in \mathcal{Q}(S)$.

(3) $\mathcal{Q}(S) \cong E(\bar{T})$.

Proof. That $(1) \Rightarrow(2)$ follows from Theorem 2.6. Next assume (2). Define $\phi$ : Q $(S) \rightarrow E(\bar{T})$ as $\phi(J)=e$ if $J \cap E(\bar{T})=\{e\}$. Then $\phi$ is clearly a bijection. That it is an isomorphism follows from Lemma 1.7. Thus (2) $\Rightarrow(3)$. That (3) $\Rightarrow(1)$ is obvious.

THEOREM 2.8. Let $S$ be a connected monoid with group of units $G$. Let e be a minimal idempotent of $S$. Let $S_{1}=\{x \mid x \in S, e x=x e=e\}$ and let $S_{2}=S_{1}^{c}$. Then

(1) $\gamma(S)=\gamma\left(S_{2}\right) \gamma(e S e)$.

(2) $\mathcal{Q}(S) \cong \mathscr{Q}\left(S_{2}\right), \alpha(S)=\alpha\left(S_{2}\right)$ and $\beta(S)=\beta\left(S_{2}\right)$.

Proof. (1) By [8, Theorem 1], eSe $\subseteq \overline{C_{G}(e)}$. By Corollary 1.20, $\gamma\left(\overline{C_{G}(e)}\right)=$ $\gamma\left(S_{2}\right) \gamma(e S e)$. Since $e$ lies in the kernel of $S, \theta(e)=1$. By Theorem 2.6, $\gamma(S)=\delta(e)$. This proves (1).

(2) Let $G, G_{2}$ denote the groups of units of $S$ and $S_{2}$ respectively. Let $T$ be a maximal torus of $G$ with $e \in \bar{T}$. Let $T_{1}=\{a \mid a \in T, a e=e\}, T_{2}=T_{1}^{c}$. By Lemma 1.3, $T_{2}$ is a maximal torus of $G_{2}$ and $E(\bar{T})=E\left(\bar{T}_{2}\right)$. Let $f \in E(\bar{T})$ and set $S_{3}=\overline{C_{G}(f)}$. By [8, Theorem 1], $e S e \subseteq f S f \subseteq S_{3}$. Then by Lemma 1.1, Theorem 2.6 and (1), $\gamma(S)=\theta_{S}(f) \gamma\left(S_{3}\right)=\theta_{S}(f) \gamma\left(S_{2} \cap S_{3}\right)^{c} \gamma(e S e)$. Also, $\quad \gamma(S)=\gamma\left(S_{2}\right) \gamma(e S e)=$ $\theta_{S_{2}}(f) \gamma\left(S_{2} \cap S_{3}\right)^{c} \gamma(e S e)$. It follows that $\theta_{S}(f)=\theta_{S_{2}}(f)$ for all $f \in E(\bar{T})$. Let $\mathscr{Q}=$ $\{J \cap E(\bar{T}) \mid J \in \mathcal{Q}(S)\}$. Since $E(\bar{T})=E\left(\bar{T}_{2}\right)$ and since $\theta_{S}(f)=\theta_{S_{2}}(f)$ for all $f \in E(\bar{T})$, we see that $\mathcal{Q}=\left\{J \cap E(\bar{T}) \mid J \in \mathcal{Q}\left(S_{2}\right)\right\}$. If $A_{1}, A_{2} \in \mathbb{Q}$, then define $A_{1}>A_{2}$ if there exist $f_{i} \in A_{i}, i=1,2$, such that $f_{1}>f_{2}$. Then by Lemma 1.7, $\mathcal{Q}(S) \cong(Q, \leqslant) \cong \mathcal{U}\left(S_{2}\right)$. In particular $\beta(S)=\beta\left(S_{2}\right)$. Clearly $\alpha(S)=|E(\bar{T})|=$ $\left|E\left(\bar{T}_{2}\right)\right|=\alpha\left(S_{2}\right)$.

THEOREM 2.9. Let $S$ be a connected monoid with group of units $G$ and let $T$ be a maximal torus of $G$. Let $S_{1}$ be a closed connected submonoid of $S$ such that $N_{G}(T) \subseteq S_{1}$. Then $\mathcal{Q}(S) \cong \mathcal{Q}\left(S_{1}\right), \alpha(S)=\alpha\left(S_{1}\right)$ and $\beta(S)=\beta\left(S_{1}\right)$.

Proof. We see that $T \subseteq N_{G}(T) \subseteq S_{1}$. So $\bar{T} \subseteq S_{1}$. Let $\mathbb{Q}=\{J \cap E(\bar{T}) \mid J \in$ थ $(S)\}$. If $A_{1}, A_{2} \in \mathbb{Q}$, then define $A_{1}<A_{2}$ if there exist $e_{i} \in A_{i}, i=1,2$, such that $e_{1}<e_{2}$. By Lemma $1.7, \mathcal{Q}(S) \cong(\mathbb{Q}, \leqslant)$. Since $N_{G}(T) \subseteq S_{1}$, we see by Lemma 1.7 that $\mathbb{Q}=\left\{J \cap E(\bar{T}) \mid J \in \mathcal{Q}\left(S_{1}\right)\right\}$. Also, by Lemma $1.7, \mathcal{Q}\left(S_{1}\right) \cong(\mathbb{Q}, \leqslant)$. Hence थ $(S) \cong$ थ $\left(S_{1}\right)$. 
THEOREM 2.10. Let $S$ be a connected monoid and let e be a minimal idempotent of $S$. Then there exists a closed connected submonoid $S_{1}$ of $S$ such that $e$ is the zero of $S_{1}$, $e \in C\left(S_{1}\right)^{c}, \alpha(S)=\alpha\left(S_{1}\right), \quad \beta(S)=\beta\left(S_{1}\right)$ and $\mathcal{Q}(S) \cong \mathcal{Q}\left(S_{1}\right)$. In particular, $\operatorname{dim} C\left(S_{1}\right) \geqslant 1$.

Proof. By Theorem 2.8, we can assume that $e=0$ is the zero of $S$. Let $G$ denote the group of units of $S$ and let $T$ be a maximal torus of $G$. By [3, Chapter IX], $\Omega=N_{G}(T) / C_{G}(T)$ is a finite group. If $u \in N_{G}(T)$, then $a \rightarrow u^{-1} a u$ is an automorphism of $T$. So $\Omega$ is a finite group of automorphisms of $T$. By Lemma 1.2, $N_{G}(T)=N_{G}(\bar{T})$ and $C_{G}(T)=C_{G}(\bar{T})$. So $\Omega$ is a finite group of automorphisms of $\bar{T}$. By Lemma 1.13, there exists a subtorus $T_{1}$ of $T$ such that $0 \in \bar{T}_{1}$ and $u^{-1} a u=a$ for all $a \in \bar{T}_{1}$ and $u \in N_{G}(T)$. Hence $N_{G}(T) \subseteq C_{G}\left(T_{1}\right)$. By [3, Theorem 22.3], $C_{G}\left(T_{1}\right)$ is a connected group. Let $S_{1}=\overline{C_{G}\left(T_{1}\right)}$. Then $N_{G}(T) \subseteq S_{1}$. By Theorem 2.9, \& $(S) \cong$ थ $\left(S_{1}\right)$. Clearly $0 \in \bar{T}_{1} \subseteq C\left(S_{1}\right)^{c}$.

Problem 2.11. Characterize the lattice $\mathcal{U}(S)$ where $S$ is a connected monoid. By Theorem 2.10, the problem reduces to the case when $S$ has zero 0 and $0 \in C(S)^{c}$.

See [9] for the following theorem.

THEOREM 2.12 [9]. Let $S$ be a connected monoid with group of units $G$ such that the maximal subgroup of the kernel of $S$ is solvable. Then the following conditions are equivalent:

(1) $G$ is solvable.

(2) $\alpha(S)=\beta(S)$.

(3) $S$ is a semilattice of archimedean semigroups.

(4) $J^{2} \subseteq J$ for all $J \in Q(S)$.

(5) For all e, $f \in E(S)$, any eigenvalue of ef is 0 or 1 .

THEOREM 2.13. Let $S$ be a connected monoid with group of units $G$. Let $J \in \mathcal{U}(S)$. Then the following conditions are equivalent:

(1) $J^{2} \subseteq J$.

(2) $\theta(J)=1$.

(3) $E(J) \subseteq \bar{B}$ for some Borel subgroup $B$ of $G$.

(4) $E(J) \subseteq \bar{B}$ for every Borel subgroup $B$ of $G$.

(5) For all $e, f \in E(J)$, any eigenvalue of ef is 0 or 1 .

Proof. (1) $\Rightarrow(2)$. Since $J^{2} \subseteq J, J$ is completely simple. So for any $e, f \in E(J)$, $e f=f e$ implies $e=f$. It follows that $\theta(J)=1$.

$(2) \Rightarrow(3)$. If $e \in E(J)$, then let $X_{e}=\left\{f \mid f \in E(S), e^{\Re} f\right\}, Y_{e}=\{f \mid f \in E(S)$, $e \varrho f\}$. If $f \in X_{e}$, then $e f=f$ and $f e=e$. If $f \in Y_{e}$, then $e f=e$ and $f e=f$. Now fix $e \in E(J)$. Let $S_{1}$ be a closed connected submonoid of $S$ of smallest possible dimension such that $X_{e} \subseteq S_{1}$. Let $S_{2}=\left\{a \mid a \in S_{1}\right.$, ae $\left.=e\right\}$. Let $f \in X_{e}$. Then there exists, by [7, Lemma 1.1], a closed connected submonoid $S_{3}$ of $S_{1}$ such that $f \in S_{3}$ and $b f=f b=f$ for all $b \in S_{3}$. So $S_{3} \subseteq S_{2}$ and $S_{3} \subseteq S_{2}^{c}$. So $f \in S_{2}^{c}$. Hence $X_{e} \subseteq S_{2}^{c}$. By the minimality of $\operatorname{dim} S_{1}, S_{1}=S_{2}$. Let $e_{1} \in E\left(S_{1}\right)$. Then by [8, Theorem 1] there exists a closed connected submonoid $S^{\prime}$ of $S_{1}$ such that $x e_{1}=e_{1} x e_{1}$ for all $x \in S^{\prime}$ and $e_{1} S_{1} \subseteq S^{\prime}$. Thus $X_{e} \subseteq S^{\prime}$. By the minimality of $\operatorname{dim} S_{1}, S^{\prime}=S_{1}$. So $x e_{1}=e_{1} x e_{1}$ 
for all $e_{1} \in E\left(S_{1}\right)$. Let $e_{1}, e_{2} \in E\left(S_{1}\right)$. Then $e_{1} e_{2} e_{1} e_{2}=e_{2} e_{1} e_{2}=e_{1} e_{2}$. Thus $E\left(S_{1}\right)^{2}$ $\subseteq E\left(S_{1}\right)$. Also $e S_{1} e=\{e\}$. Thus the hypothesis of Theorem 2.12 is satisfied. Hence the group of units $G_{1}$ of $S_{1}$ is solvable. Now $G_{1} \subseteq B_{1}$ for some Borel subgroup $B_{1}$ of $G$. Then $X_{e} \subseteq \bar{B}_{1}$. Let $f \in E(J) \cap \bar{B}_{1}$. Let $T$ be a maximal torus of $B_{1}$ (and hence of $G)$ such that $e \in \bar{T}$. By Theorem 2.1, $x^{-1} f x \in \bar{T}$ for some $x \in B_{1}$. Then $e, x^{-1} f x \in$ $E(\bar{T}) \cap J$. Since $\theta(J)=1, e=x^{-1} f x$. So $X_{f}=x X_{e} x^{-1} \subseteq x \bar{B}_{1} x^{-1}=\bar{B}_{1}$. Thus for all $f \in \bar{B}_{1} \cap E(J), X_{f} \subseteq \bar{B}_{1}$. Since $y^{-1} E(J) y=E(J)$ for all $y \in G$, and since all Borel subgroups of $G$ are conjugate, we see that $X_{f} \subseteq \bar{B}$ for any Borel subgroup $B$ of $G$ and $f \in \bar{B} \cap E(J)$. Similarly $Y_{f} \subseteq \bar{B}$ for any Borel subgroup $B$ of $G$ and $f \in \bar{B} \cap E(J)$. Now fix a Borel subgroup $B$ of $G$. Then by Theorem 2.1, there exists $e_{1} \in E(J) \cap \bar{B}$. Let $e_{2} \in E(J)$. Then by Lemma 1.12, there exist $e_{3}, e_{4} \in E(J)$ such that

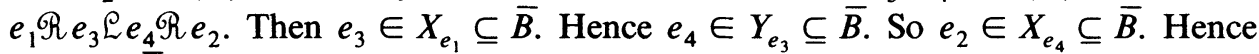
$E(J) \subseteq \bar{B}$.

(3) $\Rightarrow$ (4). This follows since $X^{-1} E(J) x=E(J)$ for all $x \in G$.

(4) $\Rightarrow$ (5) follows from Theorem 2.12 .

(5) $\Rightarrow$ (1) follows from Lemma 1.11 .

COROLlary 2.14. Let $S$ be a connected monoid with group of units $G$ and kernel $M$. Then $E(M) \subseteq \bar{B}$ for every Borel subgroup $B$ of $G$.

THEOREM 2.15. Let $S$ be a connected monoid with group of units $G$. Let e be a minimal idempotent of $S$ and let $G_{0}=\{a \mid a \in G, a e=e a=e\}$. Then the following conditions are equivalent:

(1) $\alpha(S)=\beta(S)$.

(2) $S$ is a semilattice of archimedean semigroups.

(3) $G_{0}^{c}$ is solvable.

(4) $E(S) \subseteq \bar{B}$ for some (every) Borel subgroup $B$ of $G$.

(5) For all $e, f \in E(S)$, any eigenvalue of ef is 0 or 1 .

Proof. That $(1) \Rightarrow(4)$ follows from Corollary 2.7 and Theorem 2.13. That $(4) \Rightarrow(5)$ follows from Theorem 2.12 . That $(5) \Rightarrow(2) \Rightarrow$ (1) follows from Corollary 2.7, Theorem 2.13 and [9, Proposition 21]. That (1) $\Leftrightarrow$ (3) follows from Theorems 2.8 and 2.12 .

The following is immediate from Theorem 2.1 and Corollary 2.7.

THEOREM 2.16. Let $S$ be a connected monoid such that $\alpha(S)=\beta(S)$. Then $\mathcal{Q}(S)$ is a relatively complemented lattice (in fact the lattices of all faces of a polytope in some $\left.\mathbf{R}^{n}\right)$.

Conjecture 2.17. By Theorems 2.10, 2.12, 2.15, 2.16, the following conjectures are equivalent:

(1) If $S$ is a connected monoid, then $\mathscr{Q}(S)$ is relatively complemented if and only if $\alpha(S)=\beta(S)$.

(2) If $S$ is a connected monoid with zero and group of units $G$, then $G$ is solvable if and only if $\mathcal{U}(S)$ is relatively complemented.

(3) If $S$ is a connected monoid with zero 0 and group of units $G$, if $0 \in C(S)^{c}$, and if $\mathscr{U}(S)$ is relatively complemented, then $G$ is solvable. 
We now prove Conjecture 2.17 in a special case.

THEOREM 2.18. Let $S$ be a connected monoid such that $\mathcal{Q}(S)$ is relatively complemented. If $\alpha(S) \leqslant 13$ or $\beta(S) \leqslant 7$, then $S$ is a semilattice of archimedean semigroups.

Proof. By Theorem 2.15, it suffices to show that $\alpha(S)=\beta(S)$. Assume that this is not true and find a counterexample $S$ with $\operatorname{dim} S$ minimal. Let $G$ denote the group of units of $S$. Let $e \in E(S), e \neq 1$. Then $\mathcal{Q}(e S e)$ is relatively complemented by Corollary 1.9. By Lemma 1.8, $\beta(e S e) \leqslant \beta(S)$. By Lemma 1.4, $\alpha(e S e) \leqslant \alpha(S)$. By the minimality of $\operatorname{dim} S, e S e$ is a semilattice of archimedean semigroups. Since $\alpha(S) \neq \beta(S)$, we see by Theorem 2.6 that $\theta\left(J_{0}\right)>1$ for some $J_{0} \in \mathcal{Q}(S)$. Choose $J_{0}$ maximal in $\mathcal{Q}(S)$. Let $J^{\prime} \in \mathcal{Q}(S), J^{\prime}>J_{0}$. Then $\theta\left(J^{\prime}\right)=1$. Let $T$ be a maximal torus of $G$. Then $J^{\prime} \cap E(\bar{T})=\{\xi\}$ and there exist $e_{0}, e_{0}^{\prime} \in J_{0} \cap E(\bar{T})$ such that $e_{0} \neq e_{0}^{\prime}$. By Lemma $1.7, \xi>e_{0}, \xi>e_{0}^{\prime}$. If $\xi \neq 1$, we get a contradiction to Lemma 1.10. So $\xi=1$ and $J^{\prime}=G$. Thus $G$ covers $J_{0}$.

Let $X=\{e \mid e \in E(\bar{T}), 1$ covers $e\}, Y=\{f \mid f \in E(\bar{T}), e$ covers $f$ for some $e \in X\}$. Then clearly $X \cap Y=\varnothing$. By Proposition 1.14, $J_{0} \cap E(\bar{T})=J_{0} \cap X$. If $e, e^{\prime} \in X, e \neq e^{\prime}$, then by Lemma 1.15, there exist distinct $e_{1}, \ldots, e_{k+1} \in X, f_{1}, \ldots, f_{k}$ $\in Y$ such that $e=e_{1}, e^{\prime}=e_{k+1}, e_{i}>f_{i}, e_{i+1}>f_{i}, i=1, \ldots, k$. Let $\lambda\left(e, e^{\prime}\right)$ denote the smallest such possible $k$. Let $\Omega=\left\{\left(e, e^{\prime}\right) \mid e, e^{\prime} \in X, e \neq e^{\prime}, e g e^{\prime}\right\} \neq \varnothing$. Choose $\left(e, e^{\prime}\right) \in \Omega$ with $\lambda\left(e, e^{\prime}\right)=k$ minimal. Then there exist distinct $e_{1}, \ldots, e_{k+1} \in X$, $f_{1}, \ldots, f_{k} \in Y$ such that $e=e_{1}, e^{\prime}=e_{k+1}, e_{i}>f_{i}, e_{i+1}>f_{i}, i=1, \ldots, k$. Let $e_{i} \in J_{i}$ $\in \mathcal{Q}(S), f_{i} \in J_{i}^{*} \in \mathcal{U}(S), i=1, \ldots, k$. Then with $J_{k+1}=J_{1}$ we have $J_{i}>J_{i}^{*}, J_{i+1}>$ $J_{i}, i=1, \ldots, k$. Let $\mathcal{X}=\{J \mid J \in \mathcal{U}(S), G$ covers $J\}, \mathcal{O}=\left\{J^{*} \mid J^{*} \in \mathcal{U}(S)\right.$, there exists $J \in \mathcal{X}$ such that $J$ covers $\left.J^{*}\right\}$. Then by Proposition $1.14, J_{1}, \ldots, J_{k} \in \mathcal{X}$ and $J_{1}^{*}, \ldots, J_{k}^{*} \in \mathcal{Y}$. We claim that $J_{1}, \ldots, J_{k}$ are distinct. For suppose that for some $\mu, \nu \in Z^{+}$, with $1 \leqslant \mu<\nu \leqslant k, J_{\mu}=J_{\nu}$. Then $e_{\mu} g e_{\nu}$ and therefore $\left(e_{\mu}, e_{\nu}\right) \in \Omega$. Clearly $\lambda\left(e_{\mu}, e_{\nu}\right)<k$. This contradiction shows that $J_{1}, \ldots, J_{k}$ are distinct. Next we show that $J_{1}^{*}, \ldots, J_{k}^{*}$ are distinct. For suppose $J_{\mu}^{*}=J_{\nu}^{*}$ for some $\mu, \nu \in Z^{+}$with $1 \leqslant \mu<\nu \leqslant k$. First assume $\nu>\mu+1$. Then $J_{\mu}>J_{\mu}^{*}, J_{\mu+1}>J_{\mu}^{*}, J_{\nu}>J_{\mu}^{*}$, contradicting Proposition 1.14(5). Hence $\nu=\mu+1$. But then $e_{\mu+1}>f_{\mu}, e_{\mu+1}>f_{\mu+1}, f_{\mu} \mathscr{q} f_{\mu+1}$, contradicting Lemma 1.10. Hence $J_{1}^{*}, \ldots, J_{k}^{*}$ are distinct. Next assume $k=1$. Then $e_{1} g e_{2}, e_{1}>f_{1}, e_{2}>f_{1}$. This contradicts Proposition 1.14(7). Thus $k>1$. Now assume $k=2$. Then $J_{\mu}>J_{\nu}^{*}$ for $\mu=1,2, \nu=1,2$. Hence $J_{1}^{*}=J_{1} \wedge J_{2}=J_{2}^{*}$, a contradiction. Thus $k \geqslant 3$. Since $G, J_{1}, \ldots, J_{k}, J_{1}^{*}, \ldots, J_{k}^{*}, J_{1}^{*} \wedge J_{2}^{*}$ are distinct elements of $थ(S), \beta(S) \geqslant 2 k+2 \geqslant 8$. Now let $e_{1}^{\prime}=e^{\prime} \in J_{1}$. Then $e_{1}^{\prime} \neq e_{1}$. By Proposition 1.14, $J_{i} \cap E(\bar{T}) \subseteq X, J_{i}^{*} \cap E(\bar{T}) \subseteq Y, i=1, \ldots, k$. By Lemma 1.5, there exists $f_{1}^{\prime} \in J_{1}^{*} \cap E(\bar{T})$ such that $e_{1}^{\prime}>f_{1}^{\prime}$. If $f_{1}=f_{1}^{\prime}$, then $e_{1}^{\prime}>f_{1}, e_{1}>f_{1}, e_{1} q e_{1}^{\prime}, e_{1} \neq e_{1}^{\prime}$. This contradicts Proposition 1.14(7). So $f_{1} \neq f_{1}^{\prime}$. Since $J_{2}>J_{1}^{*}$, there exists by Lemma 1.5, $e_{2}^{\prime} \in J_{2} \cap E(\bar{T})$ such that $e_{2}^{\prime}>f_{1}^{\prime}$. Suppose $e_{2}^{\prime}=e_{2}$. Then $e_{2}>f_{1}$, $e_{2}>f_{1}^{\prime}, f_{1} q f_{1}^{\prime}, f_{1} \neq f_{1}^{\prime}$, contradicting Lemma 1.10. Hence $e_{2} \neq e_{2}^{\prime}$. Continuing this process, we can find $e_{i}^{\prime} \in J_{i} \cap E(\bar{T}), f_{i}^{\prime} \in J_{i}^{*} \cap E(\bar{T}), i=1, \ldots, k$, such that for any $i=1, \ldots, k, e_{i} \neq e_{i}^{\prime}$ and $f_{i} \neq f_{i}^{\prime}$. Hence $1, e_{1}, \ldots, e_{k}, e_{1}^{\prime}, \ldots, e_{k}^{\prime}, f_{1}, \ldots, f_{k}, f_{1}^{\prime}, \ldots, f_{k}^{\prime}, f_{1} f_{2}$ are distinct elements of $E(\bar{T})$. So $\alpha(S) \geqslant 4 k+2 \geqslant 14$. Thus $\alpha(S) \geqslant 14$ and $\beta(S) \geqslant$ 8 , contradicting the hypothesis of the theorem. This proves the theorem. 
By Theorems 2.12, 2.16, 2.18 we have

THEOREM 2.19. Let $S$ be a connected monoid with zero and group of units $G$. Assume that either $\alpha(S) \leqslant 13$ or $\beta(S) \leqslant 7$. Then $G$ is solvable if and only if Q $(S)$ is relatively complemented.

REMARK 2.20. The proof of Theorem 2.18 suggests that a possible counterexample to Conjecture 2.17 has the following lattice structures:

$$
\text { थ }(S)=\left\{G, J_{1}, J_{2}, J_{3}, J_{1}^{*}, J_{2}^{*}, J_{3}^{*}, 0\right\}
$$

with the structure illustrated in Figure 1.

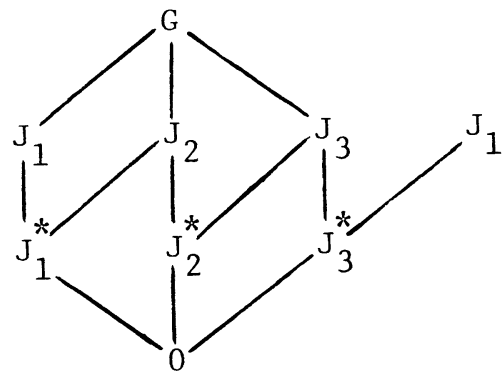

FIGURE 1

$E(\bar{T})=\left\{1, e_{1}, e_{1}^{\prime}, e_{2}, e_{2}^{\prime}, e_{3}, e_{3}^{\prime}, f_{1}, f_{1}^{\prime}, f_{2}, f_{2}^{\prime}, f_{3}, f_{3}^{\prime}, 0\right\}, e_{i}, e_{i}^{\prime} \in J_{i}, i=1,2,3, f_{i}, f_{i}^{\prime}$ $\in J_{i}^{*}, i=1,2,3$ with the structure illustrated in Figure 2.

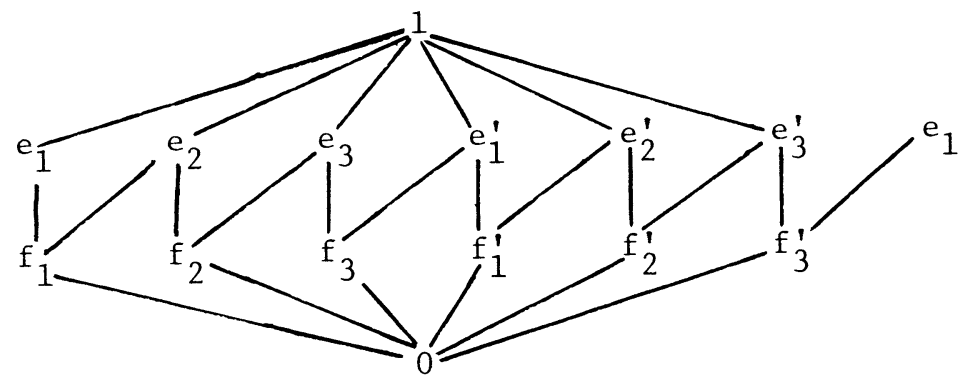

Figure 2

THEOREM 2.21. Let $S$ be a connected monoid with group of units $G$ and let $T$ be $a$ maximal torus of $G$. Let $\mathscr{V}=\{J \mid J \in \mathcal{Q}(S), \theta(J)=1\}, \Gamma=\cup\{J \cap E(\bar{T}) \mid J \in \mathcal{V}\}$. Then $\Gamma$ is a subsemilattice of $(E(\bar{T}), \cdot), \Gamma$ is the maximal semilattice image of $S$, and $\Gamma \cong(\mathcal{T}, \wedge)$.

Proof. Let $S_{\alpha}(\alpha \in \Omega)$ denote the maximal semilattice decomposition of $S$. By Tamura [10, 11], each $S_{\alpha}$ is an $\delta$-indecomposable semigroup. By [4], $\mathscr{Q}(S)=$ $\cup_{\alpha \in \Omega} \mathcal{Q}\left(S_{\alpha}\right)$. If $\alpha \in \Omega$, then let $M_{\alpha}$ denote the kernel of $S_{\alpha}$. By Theorem 2.13 and Lemma 1.21, $\mathcal{V}=\left\{M_{\alpha} \mid \alpha \in \Omega\right\}$. If $\alpha \in \Omega$, then let $e_{\alpha} \in E(\bar{T}) \cap M_{\alpha}$. Let $\alpha, \beta \in \Omega$,

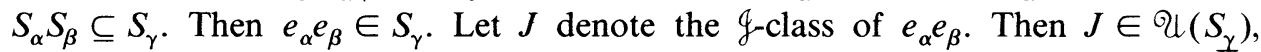
$M_{\alpha} \geqslant J, M_{\beta} \geqslant J$. Let $J^{\prime} \in \mathcal{U}(S)$ such that $M_{\alpha} \geqslant J^{\prime}$ and $M_{\beta} \geqslant J^{\prime}$. Let $h \in J^{\prime} \cap E(T)$. Then by Lemma $1.5, e_{\alpha} \geqslant h, e_{\beta} \geqslant h$. So $e_{\alpha} e_{\beta} \geqslant h$. Thus $J \geqslant J^{\prime}$. Hence $J=M_{\alpha} \wedge M_{\beta}$. By Theorem 2.6(4), $\theta(J)=1$. Hence $J=M_{\gamma}$ and $e_{\gamma}=e_{\alpha} e_{\beta}$. This proves the theorem. 
3. Central idempotents. In this section, we study special conditions on idempotents.

DEFInITION. Let $S$ be a connected monoid. Then $S$ is $E$-central if $E(S) \subseteq C(S)$. $S$ is $E$-finite if $E(S)$ is finite. $S$ is $E$-commutative if idempotents of $S$ commute with each other.

The following theorem follows from [5, Theorem 2.8].

THEOREM 3.1 [5]. Let $S$ be a connected monoid with group of units $G$ and let $T$ be a maximal torus of $G$. Then the following conditions are equivalent:

(1) $S$ is E-central.

(2) $S$ is E-finite.

(3) $S$ is E-commutative.

(4) $E(\bar{T}) \subseteq C(S)$.

(5) $E(S)=E(\bar{T})$

(6) $S$ is a semilattice of nil extensions of groups.

If $A, B$ are matrices, then let $A \oplus B$ denote $\left[\begin{array}{ll}A & 0 \\ 0 & B\end{array}\right]$. If $\mathcal{Q}, \mathscr{B}$ are sets of matrices, then let $\mathscr{Q} \oplus \mathscr{B}=\{A \oplus B \mid A \in \mathbb{Q}, B \in \mathscr{B}\}$. If $a_{1}, \ldots, a_{t} \in K$, then let $\mathcal{C}\left(a_{1}, \ldots, a_{t}\right)$ denote the set of all $t \times t$ upper triangular matrices over $K$ having $\left(a_{1}, \ldots, a_{t}\right)$ as the diagonal.

EXAmple 3.2. Let $r_{1}, \ldots, r_{t} \in Z^{+}$. Choose $\varepsilon_{i j} \in Z^{+}, 1 \leqslant i \leqslant t, 1 \leqslant j \leqslant r_{i}$. Let $w_{1}, \ldots, w_{m}$ be words in the commuting variables $X_{1}, \ldots, X_{t}$ involving each variable at least once. If $a_{1}, \ldots, a_{t} \in K$, then let $\mathscr{D}\left(a_{1}, \ldots, a_{t}\right)=\mathcal{C}\left(a_{11}^{\varepsilon_{11}}, a_{1}^{\varepsilon_{12}}, \ldots\right) \oplus \mathcal{C}\left(a_{2}^{\varepsilon_{21}}, \ldots\right)$ $\oplus \cdots \oplus \mathcal{C}\left(w_{1}\left(a_{1}, \ldots, a_{t}\right), \ldots, w_{m}\left(a_{1}, \ldots, a_{t}\right)\right)$. Let $S=\cup_{a_{1}, \ldots, a_{t} \in K} \mathscr{\mathcal { D }}\left(a_{1}, \ldots, a_{t}\right)$. Then $\bar{S}$ is a connected monoid with zero and $|E(S)|=|E(\bar{S})|=2^{t}$. So $\bar{S}$ is $E$-central. Is $S=\bar{S}$ ?

Conjecture 3.3. Let $S$ be an $E$-central connected monoid with zero. Then $S$ is isomorphic to a closed connected submonoid of a finite direct product of semigroups of the type given in Example 3.2.

THEOREM 3.4. Let $S$ be a connected monoid with group of units $G$ and let $T$ be a maximal torus of $G$. Let $\Lambda$ be a maximal chain in $E(\bar{T})$. If $\Lambda \subseteq C(S)$, then $S$ is a semilattice of archimedean semigroups.

Proof. Let $\left\{1>e_{1}>\cdots>e_{k}\right\}=\Lambda$. Since $\Lambda \subseteq C(S), \theta\left(e_{i}\right)=1, i=1, \ldots, k$. It follows by [9, Theorem 18], that $\gamma(S)=\gamma\left(e_{k}(S)\right)$. Let $G_{1}=\left\{a \mid a \in G, a e_{k}=e_{k}\right\}$. It follows from Theorem 2.8 that $\gamma\left(G_{1}^{c}\right)=1$. Hence [3, Theorem 25.2C], $G_{1}^{c}$ is solvable. By Theorem 2.15, $S$ is a semilattice of archimedean semigroups.

EXAMPLE 3.5. We give an example to show that the hypothesis of Theorem 3.4 does not imply that $S$ is $E$-central. Let

$$
\begin{gathered}
S=\left\{\left[\begin{array}{lll}
a & 0 & 0 \\
0 & b & c \\
0 & 0 & a b
\end{array}\right] \mid a, b, c \in K\right\}, \\
\bar{T}=\left\{\left[\begin{array}{lll}
a & 0 & 0 \\
0 & b & 0 \\
0 & 0 & a b
\end{array}\right] \mid a, b \in K\right\} .
\end{gathered}
$$


Then

$$
\Lambda=\left\{\left[\begin{array}{lll}
1 & 0 & 0 \\
0 & 1 & 0 \\
0 & 0 & 1
\end{array}\right],\left[\begin{array}{lll}
1 & 0 & 0 \\
0 & 0 & 0 \\
0 & 0 & 0
\end{array}\right],\left[\begin{array}{lll}
0 & 0 & 0 \\
0 & 0 & 0 \\
0 & 0 & 0
\end{array}\right]\right\}
$$

is a maximal chain in $E(\bar{T})$ and $\Lambda \subseteq C(S)$. However

$$
\left[\begin{array}{lll}
0 & 0 & 0 \\
0 & 1 & 0 \\
0 & 0 & 0
\end{array}\right] \notin C(S) \text {. }
$$

Hence $S$ is not $E$-central.

THEOREM 3.6. Let $S$ be a connected monoid with group of units $G, \alpha(S)=n$. Then

(1) If $S_{1}$ is a connected E-central submonoid of $S$, then $\left|E\left(S_{1}\right)\right| \leqslant n$. If $\left|E\left(S_{1}\right)\right|=n$, then $S_{1} \subseteq \overline{C_{G}(E(\bar{T}))}$ for some maximal torus of $T$ of $G$.

(2) If $T$ is a maximal torus of $G$, then $\overline{C_{G}(E(\bar{T}))}$ is a maximal connected $E$-central submonoid of $S$.

Proof. (1) Let $G_{1}$ denote the group of units of $S_{1}, T_{1}$ a maximal torus of $G_{1}$. Then $T_{1} \subseteq T$ for some maximal torus $T$ of $G$. Then $E\left(S_{1}\right)=E\left(\bar{T}_{1}\right) \subseteq C\left(S_{1}\right) \cap E(\bar{T})$. So $\left|E\left(S_{1}\right)\right| \leqslant n$. If $\left|E\left(S_{1}\right)\right|=n$, then $E\left(\bar{T}_{1}\right)=E(\bar{T})$ and $E(\bar{T}) \subseteq C\left(S_{1}\right)$. Then $G_{1} \subseteq$ $C_{G}(E(\bar{T}))$. So $S_{1} \subseteq \overline{C_{G}(E(\bar{T}))}$.

(2) If $e \in E(\bar{T})$, then $\bar{T} \subseteq \overline{C_{G}(e)}$. Repeated application of Theorem 2.3 yields that $C_{G}(E(\bar{T}))$ is a connected group. Hence $\overline{C_{G}(E(\bar{T}))}$ is a connected $E$-central submonoid of $S$. That it is maximal follows from (1) since $\bar{T} \subseteq \overline{C_{G}(E(\bar{T}))}$.

EXAMPLE 3.7. Let

$$
S=\left\{\left[\begin{array}{ll}
a & c \\
0 & b
\end{array}\right] \mid a, b, c \in K\right\}
$$

and let

$$
\begin{gathered}
S_{0}=\left\{\left[\begin{array}{ll}
a & 0 \\
0 & b
\end{array}\right] \mid a, b \in K\right\}, \\
S_{j}=\left\{\left[\begin{array}{ll}
a & b \\
0 & a^{j}
\end{array}\right] \mid a, b \in K\right\} \quad \text { for } j \in Z^{+} .
\end{gathered}
$$

Then $S_{i}$ is a maximal connected $E$-central submonoid of $S$ for all $i \geqslant 0$. Let $G_{i}$ denote the group of units of $S_{i}, i=0,1, \ldots$. Then $G_{0}$ is abelian, $G_{1}$ is not abelian but nilpotent, $G_{i}(i \geqslant 2)$ is not even nilpotent. Moreover $S_{i}$ is not conjugate to $S_{j}$ for $i \neq j$.

Problem 3.8. Let $S$ be a connected monoid. Study the maximal $E$-central submonoids of $S$.

THEOREM 3.9. let $S$ be a connected monoid such that for all $f \in E(S)$, the set $\{e \mid e \in E(S), e \geqslant f\}$ is finite. Then $S$ is a semilattice of archimedean semigroups.

Proof. Let $e$ be a minimal idempotent of $S$. Let $S_{1}=\{a \mid a \in S, a e=e a=e\}$. Then $S_{1}^{c}$ is $E$-finite. By Theorem 3.1, $\alpha\left(S_{1}^{c}\right)=\beta\left(S_{1}^{c}\right)$. By Theorem 2.8, $\alpha(S)=\beta(S)$. By Theorem 2.15, $S$ is a semilattice of archimedean semigroups. 
As an immediate consequence of Theorem 3.9 and [6, Theorem 3.7] we have,

THEOREM 3.10. Let $S$ be a connected regular monoid. Then the following conditions are equivalent:

(1) For all $f \in E(S)$, the set $\{e \mid e \in E(S), e \geqslant f\}$ is finite.

(2) $S$ is a Clifford semigroup.

REMARK 3.11. Theorem 3.10 is not true for connected regular semigroups (see [6, Example 4.1]).

NOTE ADDED IN THE PROOF. Theorem 2.18 is valid for any algebraic monoid with a dense group of units. Conjecture 2.17 has recently been proved by the author. See J. Algebra 73 (1981), 601-612.

\section{REFERENCES}

1. A. Borel, Linear algebraic groups, Benjamin, New York, 1969.

2. B. Grünbaum, Convex polytopes, Interscience-Wiley, New York, 1967.

3. J. E. Humphreys, Linear algebraic groups, Springer-Verlag, Berlin and New York, 1975.

4. M. S. Putcha, Semilattice decompositions of semigroups, Semigroup Forum 6 (1973), 12-34.

5. __ On linear algebraic semigroups, Trans. Amer. Math. Soc. 259 (1980), 457-469.

6. __ On linear algebraic semigroups. II, Trans. Amer. Math. Soc. 259 (1980), 471-491.

7. __ On linear algebraic subgroups. III, Internat. J. Math. Math. Sci. 4 (1981), 667-690; Corrigendum 5 (1982), 205-207.

8. __ Green's relations on a connected algebraic monoid, Linear and Multilinear Algebra (to appear).

9. __ The group of units of a connected algebraic monoid, Linear and Multilinear Algebra (to appear).

10. T. Tamura, The theory of construction of finite semigroups. I, Osaka J. Math. 8 (1956), 243-261.

11. Another proof of a theorem concerning the greatest semilattice decomposition of a semigroup, Proc. Japan Acad. Ser. A. Math. Sci. 40 (1964), 777-780.

12. __ Note on the greatest semilattice decomposition of semigroups, Semigroup Forum 4 (1972), $255-261$

Department of Mathematics, North Carolina State University, Raleigh, North Carolina 27650 